\title{
Tratamiento leXicográfico de algunos TÉRMINOS DE SILVICULTURA EN DE LA SIEMBRA Y PLANTÍOS DE ÁRBOLES (1773) DE CASIMIRO GÓMEZ ORTEGA
}

\author{
Julia Pinilla Martínez \\ Universitat de València \\ m.julia.pinilla@uv.es
}

Resumen: La ausencia de tratados completos sobre silvicultura en la España del siglo XVIII se suplió en parte con traducciones. El botánico Casimiro Gómez Ortega publicó en 1773 la traducción de la obra del agrónomo Duhamel du Monceau Des semis et plantations des bois (1760). En este artículo, estudiaremos 19 términos propios de esta disciplina desde un punto de vista lexicográfico comparando las definiciones en distintos diccionarios y en el glosario incluido en la obra origen y en la traducción. Constataremos que, a pesar de la falta de estudios publicados, gran parte de los términos ya estaban normalizados en los diccionarios de la época.

Palabras clave: Silvicultura, Historia de la traducción, Lexicografía, Siglo XVIII.

Title: Lexicographical treatment of some forestry terms in De la siembra y plantios de árboles (1773) by Casimiro Gómez Ortega.

\begin{abstract}
The absence of comprehensive treatises on forestry in 18th century Spain was partly made up for by translations. The botanist Casimiro Gómez Ortega published in 1773 the translation of the work of the agronomist Duhamel du Monceau Des semis et plantations des bois (1760). In this article, we will study 19 specific terms to this discipline from a lexicographical point of view by comparing the definitions in different dictionaries and in the glossary included in the source work and in the translation. We will note that, despite the lack of published studies, a large part of them were already standardized in the dictionaries of the time.

Keywords: Forestry, History of Translation, Lexicography, 18th Century.
\end{abstract}

\section{INTRODUCCIÓN}

La madera fue en el siglo XVIII un bien preciado por su uso en manufacturas, construcción de navíos y en la vida cotidiana. Sin embargo, los esfuerzos de las autoridades para favorecer la plantación de árboles no siempre dieron sus frutos 
pues tanto ganaderos como agricultores no supieron ver su beneficio (Gómez Ortega 1773: IX). Tampoco se contaba con tratados que contribuyeran al estudio de esta disciplina ya que las únicas especies tenidas en cuenta eran los frutales, siempre dentro de los tratados de agricultura. La excepción fue el Prólogo del tomo XIII de la obra de Antonio Ponz (1725-1792) Viaje de España publicado con el título muy explícito de Prólogo del tomo XIII del viage de España que su autor Don Antonio Ponz ha hecho imprimir separadamente. Se contienen en él varias reglas fáciles y prácticas para la siembra, plantio y multiplicación de árboles, que pueden criarse con abundancia en las Provincias del Reyno (Madrid, 1785). Esta escasez de literatura se suplió con la traducción al español del primer tratado completo de silvicultura - que se publicó en Europa- del agrónomo francés Henri-Louis Duhamel du Monceau (1700-1782) $)^{1}$ llevada a cabo por el no menos importante botánico español Casimiro Gómez Ortega (1741-1818)² (en adelante G.O.). El tratado original estaba compuesto por seis títulos en los que el autor estudió la silvicultura desde todos los ángulos. El Traité des arbres et des arbustes qui se cultivent en pleine terre (1755) describe las diferentes especies leñosas de forma enciclopédica; la Physique des arbres (1758) es un tratado de fisiología vegetal; el Traité des semis et plantations des arbres (1760) instruye sobre la importancia y el método más adecuado para el cultivo de los árboles; en De l'exploitation des bois (1764) la finalidad es extraer un beneficio de dichos cultivos; el penúltimo título enfoca la forma de transportar y trabajar la madera $D u$ transport et de la conservation et de la force des bois (1767) y finalmente aborda el cultivo de los árboles frutales Traité des arbres fruitiers (1768). Algunos de estos títulos fueron traducidos a distintas lenguas entre ellas la española, como acabamos de indicar.

Casimiro Gómez Ortega, botánico, farmacéutico, médico y escritor, a decir de Puerto Sarmiento 3 , fue el elegido por Rodríguez Campomanes - a la sazón miembro del Consejo de Castilla - para realizar la traducción (Puerto Sarmiento 1992: 275). El encargo traductor fue de tres títulos: La Physica de los árboles (1772), De la siembra y plantios de árboles (1773) y Tratado del cuidado y aprovechamiento de los montes y bosques (1773). Con estas tres traducciones, G.O. ponía al alcance de los lectores españoles una obra útil y provechosa para el desarrollo del país.

\footnotetext{
1 No hemos considerado pertinente añadir más datos biobibliográficos sobre Duhamel du Monceau por cuestiones de espacio, pues ya han sido estudiados detenidamente por Corvol (2001) y Pinilla Martínez (2008: 469-482).

2 Para mayor información sobre Gómez Ortega, véase Sempere y Guarinós (1787), Puerto Sarmiento (1992), Pinilla Martínez (2008).

3 http://dbe.rah.es/biografias/16323/casimiro-gomez-ortega.
} 
Duhamel du Monceau (en adelante D.M.) incluyó en su obra un extenso glosario Explication de plusieurs termes de Botanique \& d'Agriculture, particulièrement de ceux qui sont en usage pour l'exploitation des Bois \& des Forêts en el que se pone de relieve los términos pertenecientes a los montes y bosques. Si bien en el título no se menciona la voz 'diccionario', este cumple con todas las características: el orden alfabético de las entradas, la descripción exacta de la misma en la definición y lo que es de gran importancia para este estudio, las marcas de especialidad «Les lettres $A, B, F, J$, sont pour distinguer les termes d'Agriculture, de Botanique, de Forêts \& de Jardinage» (D.M., 1758:359). Gómez Ortega incluye la traducción del glosario conservando la marcación «Las letras $A, B, M$, J, servirán de indicar si el término es de Agricultura, Botánica, Montes, ó Jardinería» en De la siembra y plantios de árboles pensando quizá que era el tomo más apropiado por lo dicho anteriormente.

\section{ESTUDIO DE LAS ENTRADAS SELECCIONADAS}

El criterio adoptado para la selección de las entradas ha sido el siguiente: (1) que la entrada del glosario de D.M. tuviera la marca 'Forêt'; (2) que su traducción estuviera en el texto de De las siembras y plantios de árboles; (3) que dicho término traducido formara parte del glosario de G.O. con la marca 'Montes'.

Ej.:

1. Entrada en el glosario de D.M.:

GARENE (F). Bois tallis ou brousailles. Où il y a beaucoup de lapins...

2. Texto origen y traducción:

TO-A l'égard des garennes $\&$ des remises, comme ce ne sont que de petits objets, on pourra les garnir d'arbres... (Duhamel du Monceau, 1760: xxiv) TM- En punto de sotillos y matorrales para el abrigo y cría de la caza, como son cosas de menos entidad podrán poblarse de árboles... (Gómez Ortega, 1773: xxiij)

3. Entrada en el glosario de G.O.:

Sotillo (M). Bosque tallar, ó de maleza, que abunda de Conejos.

Comprobaremos asimismo si la traducción de G.O. se corresponde con la de distintos diccionarios españoles de los siglos XVIII y XIX. Hemos seleccionado entre las entradas origen unidades simples: Aunaie/Alisal; Baliveau ou Balliveau/ Resalvos; Bocage/Boscaje; Bucheron ou Boquillon/Hacheros ó carpinteros de monte; Charmé/Chamizo; Cheuron/Listón achaflanado; Cornier/Cornejal; Éclaircissement/ 
Entresaco; Éhouper/Desmochar; Essarter/Rozar; Futaie/Bosques bravos; Garene/Sotillo; Perche/Pertiga; Sépée/Cepa; Taillis/Tallares, y pluriverbales: Bois blancs/Maderas blancas; Bois vifs/Maderas vivas ó verdes; Bois vifs/Maderas vivas ó verdes; Boisde corde/ Leña de cuerda. Dado el carácter enciclopédico del glosario de DM, tanto el autor como el traductor optan por la agrupación de las entradas. Por ello, las últimas voces seleccionadas son subentradas de Bois.

Hecha la selección, hemos clasificado los términos por campos temáticos, pues es bien sabido que la voz francesa bois significa tanto madera como bosque o monte y en consecuencia la misma marcación designa subcampos diferentes.

\subsection{Bosques, montes}

En este apartado analizaremos cuatro términos referentes a los bosques y los montes. Para ello, presentaremos la entrada y artículo del texto origen (D.M.,1758), su traducción (G.O., 1773) y la presencia del término en los diccionarios españoles del siglo XVIII y mediados del $\mathrm{XIX}^{4}$. Los diccionarios consultados son el $D R A E$ en sus distintas ediciones, el Diccionario de Terreros y Pando y el de Gaspar y Roig.

La primera entrada corresponde a un tipo de monte cuyos árboles deben ser talados.

TAILlis (F). Les bois taillis sont ceux qu’on met en coupe réglée de 10, 12, 20, 30 , jusqu’à 40 ans...

Tallares (M). Véase Monte tallar.

Monte tallar. Es según la Ordenanza de Francia aquel en que los árboles no pasan de quarenta años.

Observamos en este caso que G.O. remite a una subentrada en la cual se hace mención de las normas francesas referentes a la silvicultura. En las definiciones de los dos diccionarios mencionados infra, no hemos hallado esta información sobre la normativa española.

Tanto Tallar como Monte tallar se registran en los diccionarios españoles con un sentido semejante a Taillis por primera vez en la edición del DRAE de 1803. Sin embargo, a mediados de siglo (1855), ochenta años más tarde de la publicación del glosario del traductor, Gaspar y Roig en su Diccionario ofrece la misma definición que G.O. «no pasan de cuarenta años»:

4 La información lexicográfica ha sido consultada en el Nuevo tesoro lexicográfico de la lengua española (NTLLE) de la RAE, en línea <http://buscon.rae.es/ntlle/SrvltGUILoginNtlle> $[07 / 07 / 2020]$. 
TALlaR. s,m. Monte ó porción de monte; cuya leña está en sazon para cortarse. Úsase a veces como adjetivo, como MONTE TALLAR, LEÑA TALLAR. (DRAE, 1803).

Monte Tallar: aquel en que los arboles no pasan de 40 años (Gaspar y Roig, 1855).

En Garene/Sotillo, los términos de la entrada anterior -bois tallis- se encuentran en esta definición y traducción, aunque en este caso G.O. ya no utiliza la voz monte sino bosque.

Garene (F). Bois tallis ou brousailles. Où il y a beaucoup de lapins... Sotillo (M). Bosque tallar, ó de maleza, que abunda de Conejos.

En los diccionarios consultados Sotillo es definido como diminutivo de Soto. La entrada aquí transcrita pertenece a su primera datación en el siglo XVIII:

Soto. s.m, lugar poblado de árboles, ameno y umbroso [...]. Nemus (Diccionario de Autoridades, 1739).

El Diccionario de Terreros y Pando (tomo 3,1788) incluye una entrada que resume la definición del glosario: «SOTO PARA CONEJOs. Fr. Garenne...».

Para la definición del ejemplo siguiente, G.O. recurre de nuevo a la remisión a una subentrada aunque omite la marcación.

FutAIE (F). Bois qu'on laisse parvenir à toute sa hauteur sans l'abattre.

Bosques bravos. Véase Monte.

Monte bravo. Se reputa aquel en que los árboles se dexan pasar de ciento y veinte años.

Nuestra búsqueda lexicográfica en los diccionarios españoles ha sido infructuosa en este caso pues no hemos hallado entrada o subentrada alguna de Bosque bravo o Monte bravo.

En el par Bocage/Boscaje se aprecia claramente una traducción literal:

Bocage (F). Petit bois touffu \& agréable pour les promenades.

Boscaje (M). Bosquecillo muy poblado, y deleitoso para el recreo del paseo.

En cuanto a la normalización del término boscaje, el primero en definirlo es Terreros en dos entradas (1786): 
Boscáje, multitud de arboles y enramadas. Fr. Foret, bocage

BosCÁJE, se toma también por las malezas, y enramadas de un bosque pequeño. Fr. Bocage.

Constatamos en las definiciones de Terreros la ausencia del matiz de recreo y diversión presentes en D.M. y G.O. Una hipótesis posible es la diferencia orográfica y cultural de los dos países. Francia es un país con un terreno menos montañoso y poblado de pequeños bosques que favorecían el paseo y la caza, dos de las actividades recreativas de la aristocracia y clase alta. En cambio, lo que predomina en España son los montes, espacios menos acogedores para paseos ociosos.

\subsection{Madera o leña}

Como hemos señalado, Madera es otra de las traducciones de Bois, ahora bien, no siempre es el equivalente elegido por G.O. Así vemos que para traducir Bois de corde opta por la voz leña, en cambio para la segunda entrada de este apartado Bois mort su equivalente es madera.

BoIS DE CORDE (F). A Paris, le bois de corde est formé avec des bûches depuis 6 jusquà 17 pouces de grosseur. Tout le bois à brûler doit avoir trois pieds $\&$ demi de longueur...

LEÑA DE CUERDA (M). La que sirve para lumbres, y tiene determinadas dimensiones.

A partir de la edición de 1734 del Diccionario de Autoridades, se consigna la definición de leña como «la parte de los arboles ó matas recias, que ordinariamente sirven para la lumbre...». Sin embargo, no hemos hallado entrada o subentrada alguna para leña de cuerda.

D.M. concibió la macroestructura de su glosario por agrupación de manera que determinadas entradas contienen numerosas subentradas, p.ej. bois, como ya se ha indicado anteriormente. El glosario de G.O., guarda la misma estructura origen, por consiguiente, la entrada madera se desglosa en numerosas subentradas también. Como se aprecia en el caso infra, donde ambas entradas - origen y meta- son subentradas de bois y madera respectivamente:

BOIS MORT (F). Est celui qui est desséché sur pied, ce qui differe de mort-bois, terme qui désigne des arbrisseaux de peu de valeur...

Maderas muertas (M). Arboles, que aunque parece que están vegetando, se hallan secos, y por consiguiente muertos. 
En cuanto a los diccionarios españoles, en ninguna de las obras consultadas en el NTLLE figura entrada o subentrada alguna para maderas muertas.

Finalmente, hemos seleccionado chevron para cerrar este apartado porque ya no se trata de leña o madera en bruto, sino una pieza con unas determinadas características formales, propia de un oficio.

Chevron (F). Bois équarri qui a moins de six pouces d'équarrissage.

Liston achaflanado. Véase la fig. 8.

Observamos que G.O. incluye en la entrada misma la definición al describir la forma de la pieza de madera con un solo adjetivo, remitiendo a un elemento visual presente en el tratado.

En cambio, hemos constatado que, con anterioridad a la publicación de la traducción desde la edición de 1734 del Diccionario de Autoridades y sucesivas, listón ya estaba normalizado como término de carpintería:

LISTON. Llaman los carpinteros un pedazo de tabla de dos dedos, ó mas ancho, y de poco menos grueso, de que se sirven para hacer marcos de vidrieras, para unir unas tablas con otras, y para otros usos.

\subsection{Tipos de árboles}

En este tercer subgrupo, hemos recogido y analizado el tratamiento lexicográfico de distintas denominaciones de árboles.

En el primer caso el traductor, deseoso de dar una equivalencia al mayor número posible de voces, traduce bois blancs por maderas blancas y remite a la entrada del mismo glosario árboles de ribera, con el fin de evitar la repetición de la definición.

Bois blancs (F) tels que le Saule, le Peuplier, le Tilleul, qu'on employe à des ouvrages de moindre conséquence.

Maderas blancas (M). Las de los árboles de Rivera. Véase esta voz.

ÁRBOLES DE RIBERA (M). Los que por su naturaleza vienen con particularidad en las orillas del agua y crian madera blanca y fofa por lo regular, como el alamo blanco, y negro, el Sauce, el Aliso, \&c.

El sintagma maderas blancas no se encuentra normalizado en la lexicografía de la época. Tampoco lo está maderas vivas ó verdes, traducción de bois vifs cuyas entradas hemos transcrito infra. Si bien cabe destacar desde un punto de vista léxico, el uso del par sinonímico vivas ó verdes, reflejo de inestabilidad denominativa. 
BoIs Vifs (F) est celui qui est en état de vigueur \& d'accroissement. MADERAS VIVAS Ó verdes (M). Árboles que están aún vegetando.

En el tercer ejemplo, se observa en la definición que el imperativo «on est obligé» se convierte explícitamente en imperativo legal en la traducción «por ordenanza $\gg$.

Baliveau ou Bailliveau (F). Jeune arbre au-dessous de quarante ans, qu'on est obligé de réserver dans les coupes.

Resalvos (M). Árboles nuevos de menos de quarenta años, que por ordenanza deben de dexarse en pie en las cortas de los tallares.

Resalvos, equivalente de Baliveau, figura por primera vez en el Diccionario de Terreros. No debe sorprendernos pues no es ningún secreto que para conformar su valiosa obra Terreros visitó talleres y consultó a artesanos, así como a obreros. Esto se pone de manifiesto en esta definición cuya fuente son los forestales, no las ordenanzas.

Resalvo, llaman los que cortan y cuidan de montes al árbol que reservan, quitándole los arbolitos inmediatos, que le desubstancian.

El par Charmé / Chamizo no presenta, al parecer, dificultad alguna al traductor:

ChARmÉ (F). Bois charmé, terme qui indique les arbres qu'on a fait mourir par malice: voyez Bors.

Chamizo (M). El árbol medio quemado, ó chamuscado por dañadores, ó por incendio general del Monte.

La voz chamizo se incorpora al Diccionario de Autoridades (1729) como una variedad diatópica «es voz de Galicia» que desaparecerá en las siguientes ediciones donde se sustituye por «en algunas partes».

Chamizo. s.m. Leño medio quemado. Es voz usada en Galicia (Diccionario de Autoridades, 1729).

Chamizo. s.m. En algunas partes se llama así el tizon, ó leño medio quemado (DRAE, 1780).

Nos ha parecido interesante concluir este apartado con la traducción de cornier porque hemos observado que cornejales tiene aquí una acepción diferente 
a las que se hallan en los diccionarios consultados, aunque con un elemento común: la forma en ángulo.

Cornier (F). On appelle pieds corniers, de grands arbres marqués pour indiquer les bornes d'une vente ou étendue de bois. Ils sont marqués par autorité de Justice.

Cornejales (M). Arboles de límite que se hallan en los ángulos salientes de un quartel de corta.

Si bien cabe destacar la omisión en la traducción en la traducción de la parte referente a la autoridad judicial «ils sont marqués par autorité de Justice».

La definición del Diccionario de Autoridades de 1729 se mantendrá sin apenas cambios hasta 1846 en que Vicente Salvá (1786-1849) incluyó en su Diccionario una nueva acepción relacionada con los montes.

Cornejal. s.m. La punta o esquina de alguna cosa: como una heredad, colchón, \&c. y lo mismo que Cornijál (Diccionario de Autoridades, 1729).

Cornejal. m. Cornijal. [Monte o terreno poblado de cornejos] (Vicente Salvá, 1846).

\subsection{Métodos de trabajo}

Cuidar los montes y bosques significaba asimismo realizar ciertas tareas específicas. En este cuarto apartado, hemos reunido una pequeña muestra ( 3 entradas) que analizamos a continuación.

Una de dichas tareas consistía en eliminar los árboles más débiles para favorecer el crecimiento de los demás:

ÉClaircissement (F). On dit abattre des arbres par éclaircissement, lorsqu'on n'abat que les plus foibles ou les moins venants, afin que les autres puissent mieux profiter.

Entresaco (M). Solemos decir, que hace la corta de un Monte por entresaca, quando no se cortan á hecho todos los árboles, sino yá uno en una parte, y yá otro en otra...

Aquí observamos una errata en la traducción pues no se trata de un sustantivo masculino entresaco, sino femenino como queda claro en la definición misma «por entresaca». Esta técnica debía practicarse comúnmente pues en el Diccionario de Autoridades de 1732 ya tiene entrada propia: 
Entresaca. s.f. Corte hecho en los montes, de los arboles ya viejos, ó quando están muy espesos y juntos: y porque se van sacando y cortando unos entre otros, se dixo Entresaca.

La segunda de las actividades tiene asimismo la función de limpieza de los montes como vemos infra, en la transcripción de las entradas de los glosarios:

ESSARTER (F). Est arracher tous les arbres, les arbrisseaux \& les brousailles qui couvrent un terrain...

Rozar (M). Arrancar todos los árboles, arbustos y maleza, de que está cubierto algún terreno...

Al igual que el caso anterior, rozar ya se recoge en el Diccionario de Autoridades (1737):

ROZAR. v.a. Limpiar la tierra de las matas que cría, cortándolas ó arrancándolas, para disponerla a la labor (Diccionario de Autoridades, 1737).

En el último caso de este apartado, se da la particularidad de que el traductor no utiliza la misma marcación que el autor al considerar que el término pertenece a la agricultura.

ÉHOUPeR (F). Est synonyme avec écimer; c'est couper la houpe ou la cime des arbres.

Desmochar (A). Cortar todas las ramas á un árbol, ó á un planton, de suerte que después forma en la parte superior como una cabeza, de donde brotan multitud de ramas, como se hace de quatro, ó de cinco en cinco años con los Sauces.

Este término desmochar forma parte, como los dos anteriores, del lemario del Diccionario de la RAE en su primera edición:

Desmochar. v. a. Quitar, cortar, arrancar ú desgajar la parte superior de alguna cosa, dexandola mocha: como Desmochar las reses cortándolas las hastas, desmochar el árbol desnudándole de las ramas... (Diccionario de Autoridades, 1732). 


\subsection{Oficios forestales}

Pocos son los oficios que recopila D.M., solo dos, de los cuales G.O. solo traduce uno.

Bucheron ou Boquillon (F). Ouvrier qui travaille à la coupe des bois.

Hachero, ó Carpintero de monte (M). Trabajador que se ocupa en la corta de árboles.

Abatteur (F). Ouvrier qu'on emploie à abattre les bois

Cabe destacar que la definición parece ser apropiada para ambas entradas, aunque con matices. En el caso de D.M., el bucheron corta los árboles - definición genérica- igual que el hachero. En cambio, el abatteur los tira a tierra por consiguiente existe un matiz entre ambos oficios que G.O. no tiene en cuenta.

$\mathrm{Al}$ tratarse de oficios antiguos, no es sorprendente que hachero ya figure en el Diccionario de Autoridades (1734):

HaChero. Se llama también la persona que parte leña, ó labra maderos con el hacha... (Diccionario de Autoridades, 1734).

\subsection{Varios}

Hemos nombrado este subapartado varios porque las entradas no constituyen una unidad en sí mismas. Del primer ejemplo, hay que destacar los sinónimos que G.O. utiliza para su definición. La ampliación con respecto al texto origen muestra deseo de claridad a la vez que subyace el proceso de normalización del término.

Aunaie (F). Champ planté de aunes.

Alisal, Alnetum (M). Lo mismo que Aliseda. Plantío, ó Bosque de Alisos.

En nuestro estudio, el primero en recoger esta entrada es Terreros, en 1786. El hecho de que en su artículo, como se observa infra, la traducción al francés ${ }^{5}$ coincida con la entrada del glosario completa y certifica la definición de G.O.:

Alisal, lugar de muchos alisos. Lat. Alisiarum; y como dan también muchos al aliso el nombre de olmo, el alisal se llama en Francés Aunaie (Terreros, 1786).

5 El Diccionario de Terreros incluye la traducción del lema al latín, francés e italiano. 
En este segundo ejemplo, hemos observado el respeto del traductor por el texto origen, así como el interés del mismo en informar a sus lectores de que se trata de una medida francesa.

Perche (F). Est aussi une mesure en usage pour les terres dont la longueur varie suivant les coutumes...

Pertiga (M). Medida de tierra, que en Francia consta por lo común de veinte pies de largo.

Sin embargo, al buscar la voz pertiga hemos comprobado que el término equivalente de esta acepción de perche es pértica:

Pertica. (pertica). s.f. Medida de tierra que consta de dos pasos, u diez pies geométricos... (Diccionario de Autoridades, 1737)

Pertiga. s.f. Vara larga. Es del Latino pertica... (Diccionario de Autoridades, 1737)

Nos queda la duda de si se trata de una errata, como hemos visto claramente $s u$ pra, o si el traductor desconocía esa medida, cosa poco probable pues el Diccionario de Autoridades ya la incluye en 1737.

Finalmente, cabe señalar que en el último ejemplo la traducción de G.O. no es del todo exacta:

SEPÉE (F). Touffe de plusieurs arbres qui ont été produits par une même souche...

CePA (M). La parte inferior, ó raíz de un árbol...

Observamos en la definición que Sépée ${ }^{6}$ tiene un significado plural «gavilla compuesta por varios árboles... ${ }^{7}$.

Las voces que significan «conjunto de» se crean en francés mediante el sufijo -ée, como es el caso de Cépée en el glosario. En este caso conjunto de árboles jóvenes que nacen de la misma cepa, como lo atestigua el Trésor de la langue française en su edición en línea ${ }^{8}$. El matiz de plural presente en la definición de D.M. no se recoge en la equivalencia dada por G.O. Es de todo punto improbable que el traductor no lo supiera por su dominio de la lengua gala. A nuestro parecer, no pudo resolverlo de otro modo al no hallar equivalente español.

6 Conservamos la grafía original.

La traducción es nuestra.

8 Cépée. Subs. fém. A- Touffe de jeunes tiges sortant de la souche d'un arbre coupé... 


\section{Conclusión}

El tratado de silvicultura de Duhamel du Monceau está considerado el primer tratado completo sobre silvicultura, fue traducido parcialmente a distintas lenguas y adquirió un gran reconocimiento de la comunidad científica del Setecientos. El conocimiento que ya teníamos de la obra más lo que acabamos de exponer nos decidió a estudiar las voces del glosario pertenecientes al campo de la silvicultura. Deseábamos comprobar si la terminología ya estaba normalizada en los diccionarios españoles teniendo en cuenta la poca literatura española que había en ese momento sobre esa disciplina. Las conclusiones a las que hemos llegado han estado a la altura de las expectativas. La primera de ellas es que, aun siendo voces especializadas, la mayoría están recogidas en el Diccionario de Autoridades, es decir, en la primera mitad del siglo XVIII. Pensamos que al tratarse de términos que describen una actividad antigua, pasaron a los diccionarios a través del saber popular. La segunda, que no parece que Gómez Ortega consultase en exceso las obras lexicográficas. Su conocimiento profundo de la lengua francesa y su formación científica bastaban para realizar con solvencia la traducción.

\section{Bibliografía}

Corvol, André (2001): Duhamel du Monceau: 1700-2000. Un Européen au siècle des lumières, Orléans, Académie d'Orléans. Agriculture, Sciences, Belles-Lettres et Arts.

Duhamel du Monceau, Henri-Louis (1758): La Physique des arbres, París, Chez H. L. Guérin \& L.F. Delatour.

Gaspar y Roig (1855): Diccionario enciclopédico de la lengua española..., 2 vols., Madrid, Imprenta y librería de Gaspar Roig.

Gómez Ortega, Casimiro (1773): Tratado de las siembras y plantíos de árboles y de su cultivo, Madrid, Joaquín Ibarra.

NTLLE = ReAl ACADEMIA ESPAÑola: Nuevo Tesoro Lexicográfico de la Lengua Española. En línea: <http://www.rae.es/> [07/07/2020].

Pinilla Martínez, Julia (2008): La traducción técnica y cientifica en España durante el siglo XVIII. Estudio traductológico de la obra en español de H. L. Duhamel du Monceau (1700-1782). Tesis doctoral. <https://www.tdx.cat/bitstream/handle $/ 10803 / 9844 /$ pinilla.pdf ?sequence $=1$ \&isAllowed $=y>$.

Ponz PiQuer, Antonio (1785): Prólogo del tomo XIII del viage de España que su autor Don Antonio Ponz ha hecho imprimir separadamente. Se contienen en él varias reglas fáciles y prácticas para la siembra, plantio y multiplicación de árboles, que pueden criarse con abundancia en las Provincias del Reyno, Madrid, Joaquín Ibarra. 
Puerto Sarmiento, Francisco Javier (1992): Ciencia de Cámara. Casimiro Gómez Ortega (1741-1818), el cientifico cortesano, Madrid, CSIC.

SAlvá, Vicente (1846): Nuevo diccionario de la lengua castellana, que comprende la última edición integra, muy rectificada y mejorada del publicado por la Academia Española, y unas veinte y seis mil voces, acepciones, frases y locuciones, entre ellas muchas americanas [...], París, imprenta de Don Vicente Salvá.

Sempere y Guarinós, Juan (1787): Ensayo de una biblioteca española de los mejores escritores del reynado de Carlos III, 4 vols., Madrid, imprenta Real.

Terreros y Pando, Esteban (1786-1788): Diccionario castellano: con las voces de ciencias y artes y sus correspondencias de las tres lenguas francesa, latina e italiana. Su autor el P. Esteban de Terreros y Pando, 3 vols., Madrid, viuda de Ibarra.

Trésor de la langue française en ligne. En línea: <https://www.cnrtl.fr/definition/ c\%C3\%A9p\%C3\%A9e> [15/10/2020].

Fecha de recepción: 11 de agoso de 2020

Fecha de aceptación: 23 de octubre de 2020 